\title{
Early Diagnosis of Fabry Disease in a Patient with Toe Tip Pain
}

\author{
Department of Anesthesiology and Pain Medicine, College of Medicine, Yeungnam University, Daegu, \\ Departments of *Anesthesiology and Pain Medicine, ${ }^{\S}$ Medical Genetics, Ajou University Hospital, \\ College of Medicine, Ajou University, Suwon, Department of Anesthesiology and Pain Medicine, \\ ${ }^{\dagger}$ College of Medicine, Eulji University, Eulji Hospital, Seoul, ${ }^{\ddagger}$ College of Medicine, Konyang University, Daejeon, Korea
}

Ki Bum Park, MD, Kyung Ream Han, MD*, Jae Woo Lee, $\mathrm{MD}^{\dagger}$, Seung Ho Kim, $\mathrm{MD}^{\ddagger}$, Do Wan Kim, MD*, Chan Kim, MD*, and Jung Min Ko, MD ${ }^{\S}$

Fabry disease is an X-linked lysosomal disease caused by deficiency of $\alpha$-galactosidase, in which early diagnosis may be missed due to the wide variety of clinical symptoms presenting during disease progression. A 13 year-old boy visited our pain clinic complaining of pricking and burning pain in the toe tips of both feet. Continuous epidural infusion for pain management was performed because of oral analgesics ineffectiveness. The patient underwent $\alpha$-galactosidase A (GLA) enzyme analysis based on the clinical impression of Fabry disease from pain with a peripheral neuropathic component and history of anhidrosis. He was diagnosed with Fabry disease after confirming mutation of the GLA gene through a screening test of GLA activity. Enzyme replacement therapy was initiated and pain was tolerated with oral analgesics. (Korean J Pain 2010; 23: 207-210)

Key Words:

acroparesthesia, agalsidase beta, anhidrosis, fabry disease.

Fabry disease is an $\mathrm{X}$-linked disorder characterized by deficient activity of the lysosomal hydrolase, $\alpha$-galactosidase A (GLA) [1]. Affected patients cannot effectively metabolize membrane glycosphingolipids, particularly globotriaosylceramide (GL-3), resulting in its accumulation in the vascular endothelium of various tissues [2]. Accumulation of GL-3 eventually leads to life-threatening renal, cardiac, and cerebrovascular complications, typically during the third to fifth decades of life [2,3]. The incidence of Fabry disease has been estimated to range from $1 / 40,000$ to $1 / 117,000$ live births for males [4]. In male patients with the classic phenotype, onset of symptoms oc- curs in childhood or adolescence with chronic paresthesia and episodes of severe acral and/or abdominal pain, heat intolerance, lack of sweating, and angiokeratomas [2,5]. We present a 13 year-old boy with periodic intractable pain in the toe tips of both feet. Pain was managed with continuous epidural infusion after a diagnosis of Fabry disease.

\section{CASE REPORT}

A 13 year-old boy visited our pain clinic complaining of pricking and burning pain in both toe tips. His caregiver stated that he had complained of intermittent pain in both

Received May 26, 2010. Revised July 29, 2010. Accepted July 29, 2010.

Correspondence to: Kyung Ream Han, MD

Department of Anesthesiology and Pain Medicine, Ajou University Hospital, San 5, Woncheon-dong, Paldal-gu, Suwon 442-721, Korea Tel: +82-31-219-5689, Fax: +82-31-219-5579, E-mail: painhan@hanmir.com

(c) This is an open-access article distributed under the terms of the Creative Commons Attribution Non-Commercial License (http:// creativecommons.org/licenses/by-nc/3.0/), which permits unrestricted non-commercial use, distribution, and reproduction in any medium, provided the original work is properly cited.

Copyright (c) The Korean Pain Society, 2010 
feet since he was in early elementary school. He had visited doctors of various specialties, including a psychiatrist; however, they were unable to explain the exact cause of pain. He had been prescribed analgesics such as nonsteroidal anti-inflammatory drugs for pain control. His pain had been aggravated with physical activity or hot environments so that he could not engage in physical activity at school or otherwise.

The patient initially presented with persistent pain in both toe tips, which alternated irregularly between tolerability for a couple days or weeks and intractability. In the past five months, the pain had worsened and could not be controlled with oral medications. He did not show any abnormality in his lower extremities according to sensory and motor tests. Magnetic resonance imaging (MRI) of his foot and lumbar spine was taken 2 years ago with no specific findings. Routine blood tests were normal. X-rays of the lumbar region and both feet showed osteopenia, and a bone scan revealed increased uptake of the right calcaneus. Electromyogram (EMG) and nerve conduction velocity (NCV) of his lower extremities were normal. He was considered to have peripheral neuropathy even though EMG and NCV were normal, because of the characteristics of his pain. The patient's parents also reported a history of anhidrosis. Based on pain characteristics and anhidrosis, Fabry disease was diagnosed and the patient was prescribed gabapentin (GABAPENTIN ${ }^{\circledR}$, Dong-A) for pain control.

Because toe pain persisted for 2 days despite the medication, the patient underwent epidural injection with $5 \mathrm{ml}$ of $0.3 \%$ mepivacain (MEPIVACAIN ${ }^{\circledR}$, HANA) with 50 $\mu \mathrm{g}$ of fentanyl (FENTANYL CITRATE ${ }^{\circledR}$, HANA). However, the patient was pain free for just a few hours afterward before pain recurred. Following 2 more epidural injections, continuous epidural infusion with mepivacine and fentanyl was commenced after informed consent, and he was hospitalized and evaluated for Fabry disease. The Genetics department was consulted and a test for Fabry disease was done, with GL-3 levels in plasma and urine found to be 10 times higher than normal. The GLA activity test, the hallmark enzyme test for Fabry disease, revealed no enzyme activity. Mutation analysis of the GLA gene was performed and mutation p.W349X in exon 7 was discovered, resulting in a diagnosis of Fabry disease. The patient's intermittent blurred vision and kidney function were also evaluated. No abnormalities in a 24-hour urine test were found, however turbidity in the retrolental region was found. Electrocardiogram (EKG) and echocardiogram was normal. After a screening test, his mother and younger sister were found to be carriers of the mutation, but with no major organ dysfunction. Close observation of the two was planned.

The patient experienced tolerable pain with six days of continuous epidural infusion; therefore, it was stopped. The Genetics department started enzyme replacement therapy (ERT) with $1 \mathrm{mg} / \mathrm{kg}$ of agalsidase beta (Fabrazyme ${ }^{\circledR}$, Genzyme, U.S.A) 3 weeks after Fabry disease was registered as a rare disease. During a 4-hour infusion of agalsidase beta, there were no side effects such as anaphylaxis, skin reaction, fever, chest tightness, etc. Phenytoin (PHENYTOIN $^{\circledR}$, BKW) $200 \mathrm{mg} /$ day and carbamazepine (CARBAMAZEPIN $^{\circledR}$, DAEHWA) $400 \mathrm{mg} /$ day were prescribed for pain in combination with ERT. Toe pain was consistently mild to moderate with the pain medication and ERT was performed 5 times over 8 months. Pain medication was tapered to phenytoin $100 \mathrm{mg} /$ day and carbamazepine $200 \mathrm{mg} /$ day as pain gradually decreased. Future treatment plans from the Genetics department are ERT twice a month, with regular check-ups of urine and serum GL-3 levels and kidney function every 3 months, and evaluation of EKG and echocardiogram annually.

\section{DISCUSSION}

Fabry disease has a variety of symptoms and signs, including pain attacks and life-threatening major organ failure resulting from accumulation of GL3 in the endothelium. Early treatment of Fabry disease may prevent or delay debilitating conditions, however, lack of disease awareness may delay correct diagnosis.

Ramaswami et al. [5] reported the most frequent early clinical manifestations were neurological and gastrointestinal symptoms, and were documented in about 80\% and $60 \%$ of patients, respectively. Neurological symptoms include acroparesthesia, generalized pain, altered temperature sensitivity and dyshidrosis. Abdominal pain is the most common gastrointestinal manifestation. Ries et al. [6] identified 35 pediatric Fabry disease patients (range, 1-21 years; mean, 12.6 years) in 25 families and reported that cornea verticillata was found in $73 \%$; headache, tinnitus, recurrent vertigo, diminished level of activity, fatigue, or depression were identified in 67\%; and angiokeratoma was 
found in $53 \%$ of these patients. In addition to the above symptoms, there have been reports of Fabry disease presenting with: claudication, Raynaud's phenomenon, peripheral edema, lymphoedema, depression, behavioural problems and anxiety, arrhythmia, chest pain, dyspnea, left ventricular hypertrophy, palpitations, syncope, valvular heart disease including mitral valve prolapse, systolic murmurs, and frequent infections [5].

Fabry disease might be misdiagnosed or diagnosed late because of its varying symptoms and signs. It should be suspected, however, if any of the above symptoms are present in young male patients. Family history with respect to renal, cardiovascular, and cerebrovascular events can also be very helpful in diagnosis [7]. In our case, the patient complained of intermittent pain in both feet since he was in early elementary school, and his mother experienced similar symptoms since junior high school. In addition, our patient experienced acroparesthesia, burning pain and anhydrosis.

Fabry disease is a progressive condition with lifethreatening renal, cardiac and cerebrovascular manifestations that generally develope during the third or fourth decade of life [8]. Wozniak et al. [9] collected data of hospitalized men, aged 15 to 49 years, with a first cerebral infarction identified by discharge surveillance. They described $0.65 \%$ of patients with cryptogenic strokes had previously not been diagnosed with Fabry disease. Kotanko et al. [10] reported a nationwide screening for Fabry disease of 2,480 Austrian dialysis patients in 2002 and 2003, resulting in a prevalence of $0.161 \%$. Occasionally, signs of major organ involvement have been found at an early age, as reported by Ries et al. [6]. Early diagnosis of Fabry disease is crucial in early therapeutic intervention to retard the life-threatening progression of the disease.

Ramaswami et al. [5] reported an approximately 3-year delay between the appearance of symptoms and diagnosis in a Fabry outcome survey of 11 European countries. Difficulty in diagnosis of Fabry disease is due to its rarity and variety of symptoms and signs, which can lead to misdiagnosis [6]. Of special note, acroparaethesia and dyshidrosis, as in our patient, may be the most common early presentation of Fabry disease, and practitioners should be careful when evaluating patients with these symptoms. Marchesoni et al. [11] reported on the most frequent diagnostic errors in 45 consecutive symptomatic patients with confirmed Fabry disease - the most common misdiagnosis being rheumatic fever. Sometimes, pain attacks in Fabry disease are accompanied by fever, malaise, and elevated erythrocyte sedimentation rate [12]; however, rheumatic fever is characterized by migratory polyarthritis [13]. Furthermore, they reported abdominal pain was the second most common reason for consultation in patients with Fabry disease.

ERT for Fabry disease has been available in Korea since 2002. The beneficial effects of ERT on different organs/ systems have been extensively evaluated and include improved quality of life and reduced pain severity [14]. Hoffmann et al. [15] reported the effects of ERT on pain and health related quality of life in 545 patients registered in a Fabry disease database. They described improvements in Brief Pain inventory scores after 1-2 years of treatment with agalsidase alfa. Hoffmann et al. [16] studied the prevalence and effects of ERT on abdominal symptoms associated with Fabry disease in large cohorts and concluded it reduced prevalence of gastrointestinal symptoms, particularly in children and male patients. ERT has shown a stabilization of renal function and prognosis has proven to be significantly better in cases of mild or moderate renal insufficiency from baseline [17]. Cardiac benefits have also been reported, including reduction in left ventricular mass in patients with left ventricular hypertrophy caused by Fabry disease [18]. Wraith et al. [19] evaluated the safety and efficacy of agalsidase beta in children aged 8-15 years in an open-label study, and reported that the GL-3 accumulation in dermal endothelium was effectively reduced with agalsidase beta $(1 \mathrm{mg} / \mathrm{kg})$ intravenous infusion every 2 weeks for 48 weeks. Banikazemi et al. [20] found that agalsidase beta $(1 \mathrm{mg} / \mathrm{kg})$ intravenous infusion twice a month for up to 35 months showed slower progression of major organ complications compared with a placebo group.

In conclusion, pain practitioners should be aware of the variety of Fabry disease symptoms, as early diagnosis is essential for successful treatment.

\section{REFERENCES}

1. Brady RO, Gal AE, Bradley RM, Martensson E, Warshaw AL, Laster L. Enzymatic defect in Fabry's disease. Ceramidetrihexosidase deficiency. N Engl J Med 1967; 276: 1163-7.

2. Scriver $\mathrm{CR}$. The metabolic \& molecular bases of inherited disease. 8th ed. New York, McGraw-Hill. 2001, pp 3733-74.

3. Eng CM, Fletcher J, Wilcox WR, Waldek S, Scott CR, Sillence DO, et al. Fabry disease: baseline medical characteristics of 
a cohort of 1765 males and females in the Fabry Registry. J Inherit Metab Dis 2007; 30: 184-92.

4. Meikle PJ, Hopwood JJ, Clague AE, Carey WF. Prevalence of lysosomal storage disorders. JAMA 1999; 281: 249-54.

5. Ramaswami U, Whybra C, Parini R, Pintos-Morell G, Mehta A, Sunder-Plassmann G, et al. Clinical manifestations of Fabry disease in children: data from the Fabry Outcome Survey. Acta Paediatr 2006; 95: 86-92.

6. Ries M, Ramaswami U, Parini R, Lindblad B, Whybra C, Willers I, et al. The early clinical phenotype of Fabry disease: a study on 35 European children and adolescents. Eur J Pediatr 2003; 162: 767-72.

7. Manger B, Mengel E, Schaefer RM. Rheumatologic aspects of lysosomal storage diseases. Clin Rheumatol 2007; 26: 335-41.

8. Mehta A, Ricci R, Widmer U, Dehout F, Garcia de Lorenzo A, Kampmann C, et al. Fabry disease defined: baseline clinical manifestations of 366 patients in the Fabry Outcome Survey. Eur J Clin Invest 2004; 34: 236-42.

9. Wozniak MA, Kittner SJ, Tuhrim S, Cole JW, Stern B, Dobbins $\mathrm{M}$, et al. Frequency of unrecognized Fabry disease among young European-American and African-American men with first ischemic stroke. Stroke 2010; 41: 78-81.

10. Kotanko P, Kramar R, Devrnja D, Paschke E, Voigtländer T, Auinger $\mathrm{M}$, et al. Results of a nationwide screening for Anderson-Fabry disease among dialysis patients. J Am Soc Nephrol 2004; 15: 1323-9.

11. Marchesoni CL, Roa N, Pardal AM, Neumann P, Cáceres G, Martínez $P$, et al. Misdiagnosis in Fabry disease. J Pediatr 2010; 156: 828-31.

12. Paira SO, Roverano S, Iribas JL, Barceló HA. Joint manifestations of Fabry's disease. Clin Rheumatol 1992; 11 : 562-5.

13. Madden S, Kelly L. Update on acute rheumatic fever: it still exists in remote communities. Can Fam Physician 2009; 55 :
475-8.

14. Hoffmann B, Beck M, Sunder-Plassmann G, Borsini W, Ricci R, Mehta A; FOS European Investigators. Nature and prevalence of pain in Fabry disease and its response to enzyme replacement therapy--a retrospective analysis from the Fabry Outcome Survey. Clin J Pain 2007; 23: 535-42.

15. Hoffmann B, Garcia de Lorenzo A, Mehta A, Beck M, Widmer U, Ricci R; FOS European Investigators. Effects of enzyme replacement therapy on pain and health related quality of life in patients with Fabry disease: data from FOS (Fabry Outcome Survey). J Med Genet 2005; 42: 247-52.

16. Hoffmann B, Schwarz M, Mehta A, Keshav S; Fabry Outcome Survey European Investigators. Gastrointestinal symptoms in 342 patients with Fabry disease: prevalence and response to enzyme replacement therapy. Clin Gastroenterol Hepatol 2007; 5: 1447-53.

17. Feriozzi S, Schwarting A, Sunder-Plassmann G, West M, Cybulla M; International Fabry Outcome Survey Investigators. Agalsidase alfa slows the decline in renal function in patients with Fabry disease. Am J Nephrol 2009; 29: 353-61.

18. Hughes DA, Elliott PM, Shah J, Zuckerman J, Coghlan G, Brookes $J$, et al. Effects of enzyme replacement therapy on the cardiomyopathy of Anderson-Fabry disease: a randomised, double-blind, placebo-controlled clinical trial of agalsidase alfa. Heart 2008; 94: 153-8.

19. Wraith JE, Tylki-Szymanska A, Guffon N, Lien YH, Tsimaratos M, Vellodi A, et al. Safety and efficacy of enzyme replacement therapy with agalsidase beta: an international, open-label study in pediatric patients with Fabry disease. I Pediatr 2008; 152: 563-70.

20. Banikazemi M, Bultas J, Waldek S, Wilcox WR, Whitley CB, McDonald M, et al. Agalsidase-beta therapy for advanced Fabry disease: a randomized trial. Ann Intern Med 2007; 146: $77-86$. 